\title{
APS REINSURANCE
}

\author{
By Bruno Koller, Nicole DetTwyler
}

\begin{abstract}
This paper presents a new reinsurance product, called 'Adaptive Pivot Smoothing' (APS). It is designed to reduce the variance of the risk reinsured without affecting the mean. Investment theories have provided the idea for the product.
\end{abstract}

\section{KEYWORDS}

Reinsurance, Financial Reinsurance, Smoothing.

\section{THE NEED OF A NEW REINSURANCE PRODUCT}

'Adaptive Pivot Smoothing', APS for short, is a new reinsurance product. Why do we need a new reinsurance product? Do existing products not already provide safe cover for all of the needs of insurance companies? We believe that traditional reinsurance treaties have three shortcomings in practice.

Firstly, traditional reinsurance products do not take account of the ideas of modern portfolio theory. Investors and insurance company managers alike aim to maximise the returns on their portfolios and, at the same time, minimise the volatility of the results. We have come up with a reinsurance product which does not alter the expectation of the claims distribution but which manages to reduce the variance to a level defined by the direct insurer. 'Pivot' in the acronym APS hints at this feature.

Secondly, there is more and more demand for reinsurance with potential risk of misuse. Health insurance policies spring to mind here most notably. Generally speaking, health insurance portfolios are quite homogeneous, without significant fluctuations in terms of loss load. In most cases reinsurance is not necessary. The actual problem facing health insurance companies is how to adapt premiums on time to the rapidly growing costs of health care. This is not always possible for political reasons. Consequently, insurers might be tempted to pass poor loss performance on to the reinsurer. If the reinsurance company, in turn, insists on increasing the premiums, the health insurance companies cancel their reinsurance policies. An experience rating component in our new product greatly reduces the potential for misuse of the cover. The term 'Adaptive' in APS reminds of this characteristic.

The third shortcoming often comes to light when a new insurance product is launched. In response to increased pressure from their competitors, insurance companies must develop covers and set rates with only very sketchy statistical material available sometimes. A reinsurance program would be advantageous 
which a) offered temporary financing in cases where premiums are too low and b) skimmed off profits in cases where premiums are too high, setting these funds aside to be used later on. This gives the insurance company time to analyse business trends and to adapt premiums accordingly. Our new reinsurance product allows profits and losses to be carried forward to later years - the term 'Smoothing' in APS was chosen for this reason.

\section{TRADITIONAL REINSURANCE}

In technical terms, reinsurance means splitting a risk in two. One part of the risk (the retention) rests with the insurer, while the other part is ceded to the reinsurer. The split is determined by the payment function $h(S)$, which specifies how much the reinsurer is required to pay towards every claim $S$ ( $S$ is a random variable). Thus, the insurance company carries the retention, $S-h(S)$, the reinsurer the risk $h(S)$.

The expected value of the retention distribution is

$$
E[S-h(S)]=E[S]-E[h(S)]
$$

and the expected value of the ceded distribution is

$$
E[h(S)]
$$

The variance and the standard deviation of the claims distribution of $S$ are labelled $V A R[S]$ and $S D V[S]$ respectively. The variance of the retention distribution, $V A R[S-h(S)]$ is:

$$
\begin{aligned}
V A R[S-h(S)] & =V A R[S]+\operatorname{VAR}[-h(S)]+2 \cdot \operatorname{COV}[S,-h(S)] \\
& =V A R[S]+V A R[h(S)]-2 \cdot \operatorname{COV}[S, h(S)] .
\end{aligned}
$$

Writing $K$ for the correlation coefficient we get:

$$
V A R[S-h(S)]=V A R[S]+\operatorname{VAR}[h(S)]-2 K \cdot S D V[S] \cdot S D V[h(S)] .
$$

If we make the obvious assumption that the correlation between $S$ and $h(S)$ is positive, the value $K$ must be between 0 and 1, which enables us to make the following estimate:

$$
(S D V[S]-S D V[h(S)])^{2} \leq V A R[S-h(S)] \leq V A R[S]+V A R[h(S)] .
$$

If there is complete linear correlation $(K=1)$, the following equations apply:

$$
\begin{gathered}
V A R[S-h(S)]=(S D V[S]-S D V[h(S)])^{2} \\
S D V[S-h(s)]+S D V[h(S)]=S D V[S] .
\end{gathered}
$$

Thus, the reduction of the variance can be achieved most effectively by means of a linear payment function! 
The situation of the cedent buying reinsurance can be quantified by

$$
S-P[S]-h(S)+Q[h(S)]
$$

here $P$ represents the premium income and $Q$ the reinsurance premium. If we assume that $Q[h(S)]>E[h(S)]$, consequently

$$
E[S-P[S]-h(S)+Q[h(S)]]>E[S-P[S]] .
$$

In other words, reinsurance increases the mean burden on the cedent.

However, if reinsurance is unable to lower the mean burden at all, it is difficult to see how a payment function $h$ can be used with $E[h(S)]>0$. (Common to all traditional forms of reinsurance - proportional and non-proportional - is $E[h(S)]>0$.) This merely amounts to an exchange in premiums and losses without giving rise to any economic benefit. It would be much wiser for the cedent to agree on a payment function with $E[h(S)]=0$.

The reinsurer obviously cannot be expected to lower the cedent's burden. The benefit offered by reinsurance is that it brings about a reduction in volatility or, if volatility is measured in terms of variance, a precisely quantified reduction of the variance. Consequently, the cedent should be asking the reinsurer to reduce the variance of the claims distribution by $x$ per cent; the service provided by the reinsurance company can then be assessed accurately.

To sum up, an insurance company would be best advised to take out a reinsurance policy in which $E[h(S)]=0$ and $S D V[h(S)]=c \cdot S D V[S]$, where $0<c<1$.

This view of reinsurance is very much in accordance with modern portfolio theory. The investor is aiming to maximise expected returns, whilst at the same time minimise volatility. The risk manager at an insurance company endeavours to achieve precisely the same effect, stabilising profits on a high level with small fluctuations.

\section{APS REINSURANCE}

The above analysis clearly shows that the new product should display a linear payment function, whereby the ceded distribution should have an expected value of zero.

Assuming a linear payment function of

$$
h(S)=c \cdot(S-E[S]), \text { where } 0<c<1,
$$

we can derive the following relations:

$$
\begin{gathered}
E[h(S)]=0 \\
E[S-h(S)]=E[S] \\
V A R[h(S)]=c^{2} \cdot V A R[S] \\
V A R[S-h(S)]=(1-c)^{2} \cdot V A R[S] .
\end{gathered}
$$


The point $(E[S], 0)$ where the linear payment function intersects the $\mathrm{x}$-axis has been called the 'Pivot' in order to emphasize that both losses and profits are affected by reinsurance. If the claims ratio is higher than the pivot, the reinsurer pays the cedent. However, if it is smaller, the cedent pays the reinsurer.

It is crucial to the success of this design that the payment function intersects the $x$-axis at the expected value of the claims distribution. Of course the 'true' expected value is hardly ever known. What is more, the expected value can shift with time. Therefore, we have to consider a mechanism which automatically adapts the payment function, or to be more precise the pivot, to current claims experience. This is a vital component of APS Reinsurance.

The adaptation mechanism must be defined in the treaty to ensure that the cedent and the reinsurer do not disagree on the 'correct' pivot. If the adaptation mechanism is to work properly, it must be unambiguous, simple and efficient.

It is important to understand that we are faced here with a forecast problem. We have to forecast the pivot for the coming financial year - not assess the pivot for the previous period. The cedent needs to know the exact terms of the reinsurance treaty in advance. Section 5 examines an adaptation mechanism. based on credibility; other methods are possible and may be even better, depending on the situation.

With our choice of the payment function, the expected value of claims being ceded is zero. Therefore the premium, calculated as expected claims plus loading', will be much lower than the premium for traditional reinsurance products. The choice of the premium calculation principle needs to be given very careful consideration. The 'expected value principle', for instance, is pointless here. The variance principle or the standard deviation principle are possible. However, we recommend that the principle of zero utility be used (cf. GERBER (1979), page 67) because also higher moments should be taken into account, given the dominance of the loading.

\section{IMPLEMENTATION}

APS Reinsurance is based on the claims ratior of a certain portfolio. The claims ratio for financial year $t$ is defined by

$$
r_{1}(t)=-S(t) / P(t), \text { where } S(t) \leq 0, P(t)>0 .
$$

$S(t)$ represents the claims during financial year $t$, and $P(t)$ represents the premium income during the same period. When calculating $r_{1}(t)$, premium income, claims and claims reserve must be allocated to the correct year.

The payment function $h$ becomes a function of claims ratio $r_{1}$ :

$$
h\left(r_{1}(t)\right)=a \cdot\left(r_{1}(t)-E\left[r_{1}(t)\right]\right), \text { where } 0<a<1 .
$$

The parameter $a$ is referred to as the smoothing factor. It defines the reduction in standard deviation brought about by the reinsurance. 
The claims ratio after reinsurance, $r_{2}$, becomes

$$
r_{2}(t)=r_{1}(t)-h\left(r_{1}(t)\right)=(1-a) \cdot r_{1}(t)+a \cdot E\left[r_{1}(t)\right]
$$

$r_{2}$ lies between $r_{1}$ and $E\left[r_{1}(t)\right]$, thus damping the volatility of $r_{1}$.

At the end of the financial year $t$, the claims ratio $r_{2}(t)$ is calculated from $r_{1}(t)$ on the basis of the above equation. $r_{2}$ serves to calculate the amount of money to be paid, called the smoothing benefit $L(t)$ :

$$
L(t)=-r_{2}(t) \cdot P(t)-S(t) .
$$

It is important to understand that if $L$ is positive (the observed claims ratio being higher than the expected value), the reinsurer pays benefit to the cedent. Otherwise, the cedent pays the reinsurer. In practice, the reinsurer generally incorporates (upper and lower) limits to the smoothing benefit into the terms of the contract.

The target of the smoothing procedure, i.e. the pivot $b(t)$, is the expected value of the random variable $r_{1}(t)$. Therefore, the equation for the claims ratio $r_{2}$ can also be written as:

$$
r_{2}(t)=(1-a) \cdot r_{1}(t)+a \cdot b(t)=b(t)+(1-a) \cdot\left(r_{1}(t)-b(t)\right) .
$$

At the start of the reinsurance, the pivot is fixed on the basis of calculations or observations. Subsequently the pivot is automatically adapted on the basis of the most recent claims experience. One obvious choice for the adaptation mechanism is to use a credibility approach:

$$
b(t+1)=g(t) \cdot r_{1}(t)+(1-g(t)) \cdot b(t)
$$

$g(t)$ is called the credibility weight, or credibility for short. The advantage of this type of formula is that it incorporates all of the past experience, though with an exponential decrease in weighting. $g$ is generally regarded independent of time. The above approach, in which weighting is time-related, is referred to as 'adaptive exponential smoothing' in time series analysis (cf. ABRAHAM and Ledolter (1983), page 377). In section 5 we propose a formula for $g(t)$ which has proved its worth in simulations of business procedures.

Thanks to the adaptation mechanism, profits and losses can be carried forward in APS Reinsurance. The smoothing benefit is carried over to the next year either in full or in part, and is offset against earlier payments. When the treaty is terminated, the accrued balance is paid back in full or in part over a predefined period of time. The adaptation mechanism guarantees that the (positive or negative) balance cannot grow without limits. Technically speaking, the profits and losses carried forward belong to the insured party and attract interest (positive or negative).

Depending on whether the balance is carried forward and/or settled at the end in full or only in part, APS Reinsurance becomes more or less a financial reinsurance. 
In some cases it may be advisable to ask the cedent to pay a deposit at the start of the year. The deposit is offset against the smoothing benefit at the end of the year.

The ceded distribution can be calculated from the distribution of the aggregate claim amount and the payment function. If profits and losses are not being carried forward, the calculation of the premium is based on this distribution. If profits and losses are carried over and the final balance is not fully settled, we have to deal with a sum of random variables and the premium calculation is based on the folded distribution. Due account must be taken of the precise terms and conditions regarding termination of the treaty.

If the profits and losses are carried over in full and the final balance fully settled, there is no longer any technical risk. In this event, commissions should be requested instead of premiums. The commissions should be based on the difference between the premiums for risk $r_{1}$ and risk $r_{2}$ (cf. section 5), as this difference reflects the benefit which the cedent derives from the reinsurance.

\section{EXAMPLE}

In this section we will make assumptions about the claims distribution, specify the premium calculation principle and discuss a pivot adaptation mechanism.

We regard the claim $S$ as being stochastic, whereas we view the cedent's income from premiums $P$ as being deterministic. Let the claims be normally distributed. (Other distributions might be more realistic, but the normal distribution is better suited to illustrate the important points.) The standard deviation of the normal distribution is fixed, whereas the expected value is a function of time.

It follows that the claims ratio $r_{1}$ is also normally distributed. Thanks to the linear payment function, the ceded distribution of $r_{1}-r_{2}$ and the retention distribution of $r_{2}$ are also normal with:

$$
\begin{gathered}
E\left[r_{2}\right]=E\left[(1-a) \cdot r_{1}+a \cdot E\left[r_{1}\right]\right]=E\left[r_{1}\right] \\
E\left[r_{1}-r_{2}\right]=E\left[r_{1}-(1-a) \cdot r_{1}-a \cdot E\left[r_{1}\right]\right]=0 \\
S D V\left[r_{2}\right]=S D V\left[(1-a) \cdot r_{1}+a \cdot E\left[r_{1}\right]\right]=(1-a) \cdot S D V\left[r_{1}\right] \\
S D V\left[r_{1}-r_{2}\right]=S D V\left[r_{1}-(1-a) \cdot r_{1}-a \cdot E\left[r_{1}\right]\right]=a \cdot S D V\left[r_{1}\right] .
\end{gathered}
$$

The premium calculation principle which we select is the exponential principle (cf. Gerber (1979) for instance) with risk aversion $d$. Instead of defining the utility of money, we regard it as a function of the claims ratio. Consequently, when we apply the exponential principle we obtain a premium rate instead of a premium amount. The premium rate is then calculated as

$$
Q[r]=\log \left(E\left[e^{d r}\right]\right) / d, \text { where } d>0 .
$$

Our approach is theoretically not quite correct. To a company sums of money are important, not ratios. Nevertheless, it provides us with a reinsurance premium 
which is proportional to the cedent's premium income the simplest approach in practice. It is advisable to adapt the risk aversion $d$ depending on the size of the reinsured portfolio.

For a normal distribution the above formula becomes

$$
Q[r]=E[r]+0.5 \cdot d \cdot V A R[r] .
$$

Consequently, the reinsurer has to charge the following premium rate for the APS Reinsurance product:

$$
Q\left[r_{1}-r_{2}\right]=0.5 \cdot d \cdot a^{2} \cdot V A R\left[r_{1}\right]
$$

and the APS premium is calculated as $Q\left[r_{1}-r_{2}\right] \cdot P$.

If we compare the APS premium rate with the rates for $r_{1}$ and $r_{2}$,

$$
\begin{gathered}
E\left[r_{1}\right]+0.5 \cdot d \cdot V A R\left[r_{1}\right] \\
E\left[r_{1}\right]+0.5 \cdot d \cdot(1-a)^{2} \cdot V A R\left[r_{1}\right],
\end{gathered}
$$

we see that the value $Q\left[r_{1}-r_{2}\right]$ is smaller than the difference between the two rates. In other words, the benefit which the cedent derives from the reinsurance product is higher than the reinsurance premium.

We discuss the following pivot adaptation mechanism:

$$
b(t+1)=g(t) \cdot r_{1}(t)+(1-g(t)) \cdot b(t) .
$$

It certainly makes sense that $g$ is a function of $r_{1}, b$ and $S D V\left[r_{1}\right]$ : $g\left(t ; r_{1}(t), b(t), S D V\left[r_{1}\right]\right)$. The greater the difference between $r_{1}(t)$ and $b(t)$, the higher the probability that the old estimate $b(t-1)$ is obsolete and that greater weighting should be allocated to the claims ratio currently observed: the credibility $g(t)$ should increase. Furthermore, the difference between the old pivot and the new observed value is all the more significant if the standard deviation is low: the credibility $g(t)$ should also increase in this case. The simplest quantity which meets these requirements is

$$
\left|r_{1}(t)-b(t)\right| / S D V\left[r_{1}\right] .
$$

The above expression can have values between 0 and infinity, while $g(t)$ must be between 0 and 1 . Therefore, we need a strictly increasing function which maps the positive real numbers on the interval $[0,1]$.

The above expression has a standard normal distribution; remember that $b$ is the expected value of $r_{1}$. An obvious choice for the required transformation function is the probability of the interval

$$
\left(-\left|r_{1}(t)-b(t)\right| / S D V\left[r_{1}\right],+\left|r_{1}(t)-b(t)\right| / S D V\left[r_{1}\right]\right) .
$$

The credibility is then calculated as

$$
g(t)=2 \cdot\left[S N D\left(\left|r_{1}(t)-b(t)\right| / S D V\left[r_{1}\right]\right)-0.5\right],
$$

where $S N D$ stands for the standard normal distribution function. 


\section{APPLICATION OF APS REINSURANCE}

APS Reinsurance is generally suitable for covering portfolios which entail a relatively high frequency of claims. Health insurance portfolios typically fulfill these requirements. If, on the other hand, claims are very rare like for instance in catastrophe reinsurance, the adaptation mechanism will not provide any suitable results.

APS Reinsurance is specially designed to suit sectors which are faced with increasing claims costs. The claims ratio typically rises over a period of a few years until the premiums are adapted. The claims ratio then drops and the cycle starts all over again. APS Reinsurance is ideal as a smoothing instrument in a case such as this.

The product can also play an important role when a new product is being set up. Statistics often prove to be unreliable at the start. With the aid of APS Reinsurance, results which are poor or which are (overly) good during those first few years can be carried over to later years, giving the cedent time to adapt the premiums.

Quota share reinsurance achieves the same goal as APS Reinsurance: it reduces the variance of the aggregate claims distribution to a certain percentage. However, the cedent is required to pay a considerable share of its premiums to the reinsurer. This is most of the time undesirable, so quota share reinsurance is no longer common.

Surplus Reinsurance also reduces the variance of the aggregate claims distribution. But the extent of the reduction is very difficult to calculate, whereas in APS Reinsurance the reduction percentage is part of the treaty. Stop loss reinsurance, which also serves to reduce the variance, has the disadvantage of a heavily loaded premium. This can be avoided in APS Reinsurance by implementing it as a financial reinsurance.

In spite of having a linear payment function, APS Reinsurance is not a proportional reinsurance. Proportional reinsurance is defined as being an agreement where the cedent and the reinsurer share premiums and losses in the same proportion. It is important to understand why this is not the case. APS Reinsurance does not carry losses - it reduces the volatility!

One of the advantages of APS is that it can be turned into a financial reinsurance. The dilemma with financial reinsurance is to provide enough risk exposure so that the supervising authorities class the treaty as an insurance and not as a banking transaction. If treated as a banking transaction, the smoothing effect disappears because of the profits and losses carried over show up in the balance sheet. There are a number of ways of including more risk, which have been discussed above (section 4). Another possibility not yet mentioned would be to limit the smoothing benefit and cover the excess by a conventional stop loss reinsurance; the stop loss reinsurance would then be part of the APS treaty. 


\section{REFERENCES}

Abraham, B. and LfDOlTFr, J. (1983) Statistical Methods for Forecasting. John Wiley \& Sons, New York.

Gerber, H.U. (1979) An Introduction to Mathematical Risk Theory. S.S. Huebner Foundation Monograph 8, University of Pennsylvania, Philadelphia.

\section{BRUNO KOLLER \\ Dinkelbergstr. 21 \\ CH-4I25 Riehen}

Nicole DetTwyler

Petersgraben 21

CH-405I Basel 